\title{
A response to Letter to the Editor titled: Valproic acid for myoclonic epilepsy in POLG1 carriers can be fatal
}

Dear Dr. Josef Finsterer,

Thank you for your careful and thorough reading of our article and for the valuable comments.

We agree that valproic acid is harmful in the mitochondrial disease and we want to add that the described case is currently being prosecuted as medical malpractice.

This patient was born as a dizygotic twin and she developed slightly worse than her brother. Despite this fact she was not diagnosed because her worse development was attributed to the twin pregnancy. When seizures started, valproic acid (VPA) was given at a regional neurological department because of abnormal EEG results. Seizures occurred 2-3 times a day, and treatment with a target dose of $350 \mathrm{mg}$ 0-350 mg of VPA was started. VPA serum level was $84.10 \mu \mathrm{g} / \mathrm{ml}(\mathrm{N}: 50-100 \mu \mathrm{g} / \mathrm{ml})$. The liver tests were normal at that time and her brain MRI did not show abnormalities suggesting mitochondrial disorders. MRI both without and with contrast in T1, T2, FLAIR, DWI, PWI modalities was carried out. MRS was not performed. Cerebrospinal fluid had not been tested. Within a few weeks, she started experiencing abdominal pain and was diagnosed with pancreatic damage on ultrasound examination. Mitochondrial disease was not suspected in the regional hospital. Liver damage was the next symptom that occurred over the following week. As her condition deteriorated dramatically she was sent to the highest reference centre for consideration of a possible liver transplantation. Unfortunately, the child died there within 4 days due to multiorgan insufficiency. It was surprising that at the time pancreatic injury was identified, liver failure parameters (ALAT and coag- ulation tests as well as ammonia) were still normal and worsened only a few days before death. Her illness lasted around 3 months. A genetic test revealing the POLG mutation was performed as part of the investigation towards the causes of the problem. We only had information that the parents were healthy, as were her twin brother and 13-year-old brother.

We want to emphasize that the results of genetic tests were obtained after the child's death and only then was it possible to make a diagnosis.

Alpers-Huttenlocher core symptoms - seizures, developmental regression and liver failure may be caused not only by POLG mutations, but also by several other gene mutations which caused mtDNA depletions, among other by TWNK, DGUOK, MPV17, $R R M 2 B$ and other. Therefore, in the literature on mitochondrial diseases and between specialists in this field of expertise, the term syndrome is used in regards to Alpers-Huttenlocher and not disease, which as a term is reserved for monoetiological disease entities.

The diagnostics of mitochondrial diseases is difficult and complex, and due to the low specificity of symptoms, these diseases are often diagnosed too late. That is why, among other things, we decided to show this case. However, the main reason for describing this case in Folia Neuropathologica was the interesting neuropathological picture and that is what we wanted to highlight.

Best regards, Sylwia Tarka, MD, PhD Department of Forensic Medicine Medical University of Warsaw, Warsaw, Poland 